
07.05.2020

Published: 15.06.2020

Keywords

hand ultrasound, hand anatomy, fingers tendon, hand tendon

\section{Submitted: \\ 26.03.2019 \\ Accepted: \\ Ultrasound anatomy of the fingers: flexor and extensor system with emphasis on variations and anatomical detail}

\author{
Michel De Maeseneer ${ }^{1,2}$, Jie Meng ${ }^{3}$, Stefaan Marcelis ${ }^{4}$, Tjeerd Jager ${ }^{5}$, \\ Steven Provyn ${ }^{6}$, Maryam Shahabpour ${ }^{7}$
}

${ }^{1}$ Vrije Universiteit Brussel, Brussels, Belgium

${ }^{2}$ Department of Radiology, Radiologie Buggenhout, Buggenhout, Belgium

${ }^{3}$ Department of Ultrasound, Jilin University, Changchun Jilin, China

${ }^{4}$ Department of Radiology, Sint Andries Ziekenhuis SAT, Tielt, Belgium

${ }^{5}$ Department of Radiology, ASZ, Aalst, Belgium

${ }^{6}$ Department of Anatomical Research and Clinical Studies, Vrije Universiteit Brussel, Brussel, Belgium

${ }^{7}$ Department of Radiology, Universitair Ziekenhuis Brussel, Brussels, Belgium

Correspondence: Michel De Maeseneer, MD, Department of Radiology, Laarbeeklaan 101, 1090 Jette,Belgium; e-mail: Michel.demaeseneer@uzbrussel.be

DOI: $10.15557 / \mathrm{JoU} .2020 .0020$

\begin{abstract}
Many anatomical details and variants occur in the finger tendons and soft tissue structures. These may lead to misdiagnosis if the radiologist is not well aware of them. We discuss the midhand extensor tendons, dorsal hood, junctura tendinea, conjoint tendons, transverse retinacular ligament, triangular ligament as well as central and distal slip anatomy and ultrasound correlation. The dorsal hood is an important structure to center the tendons at the midportion of the MCP heads, and the sagittal bands are its main components. Two tendons are present at the second digit, and two or more at the fifth digit. The extensor mechanism is anatomically interrelated with the palmar lumbricals and interosseous tendons. At the palmar side, the flexor superficialis and profundus tendons show varying relationships along the finger. The flexor profundus passes through an opening in the flexor superficialis. We also discuss the chiasma crurale, ridges at the flexor superficialis insertions and bifid flexor profundus tendon. Although a typical distribution of annular pulleys can be observed, many variants may be present of which we address some. The volar plate is a midline fibrocartilaginous meniscus attached proximally to the well-identifiable checkrein ligaments and distally to the base of the phalanges. Knowledge of these details and variations allows for better understanding of the finger and hand ultrasound.
\end{abstract}

\section{Introduction}

Many variations can be observed in the anatomy of the extensor and flexor tendon system of the hand ${ }^{(1-4)}$. These variations may involve the extensor tendons at the wrist and midhand level. Variations also occur in the tendons and pulleys at the flexor side. These variations present potential pitfalls at ultrasound (US) and radiologists should be aware of them to avoid misdiagnosis. The finger extensor and tendon system is complex and many details are not well known by radiologists.
Knowledge of this complex anatomy is also important to correctly interpret ultrasound images. Our aim is to provide an overview of clinically relevant variations, complex areas, and anatomical details that radiologists should be aware of. We address the anatomy and variants at the level of the midhand and dorsal hood. Next, we address the complex extensor system at the finger. At the volar side, we review the complex anatomy of the flexor system, anatomical details and variations of the flexor superficialis and profundus tendons, variations in the pulley system, and the anatomy of the volar plate 


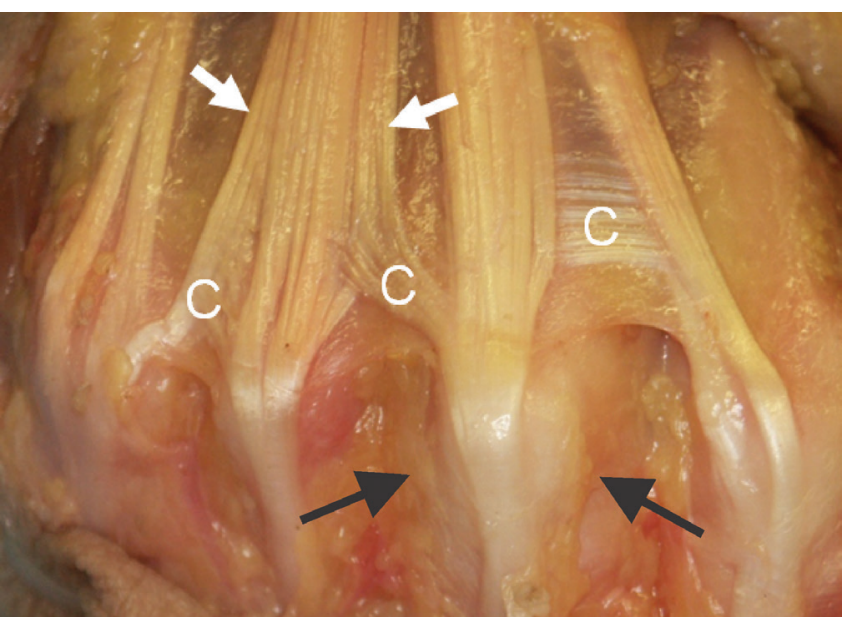

Fig. 1. Distal midhand. Note the broadening of extensor tendons at midhand (arrows) with pseudosplitting. Also, the connexus can be observed $(C)$ between the extensor tendons. At the level of the MCP joints, the extensor tendons are kept in place by the sagittal bands (dorsal hood, black arrows)

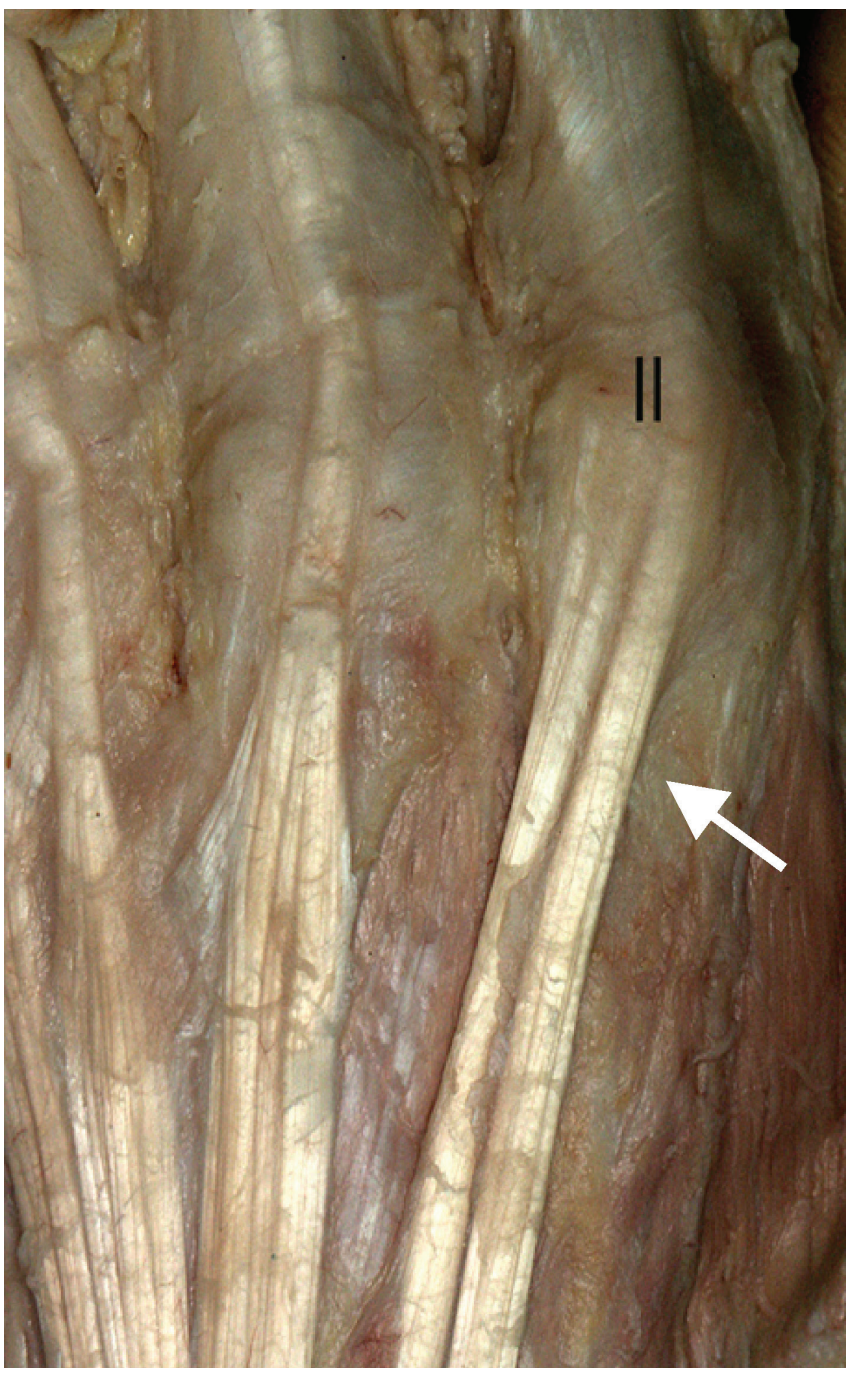

Fig. 2. Dissection of the 2 nd row on the level of metacarpals of the left hand dorsally. Note the presence of 2 tendons for this finger (arrow)

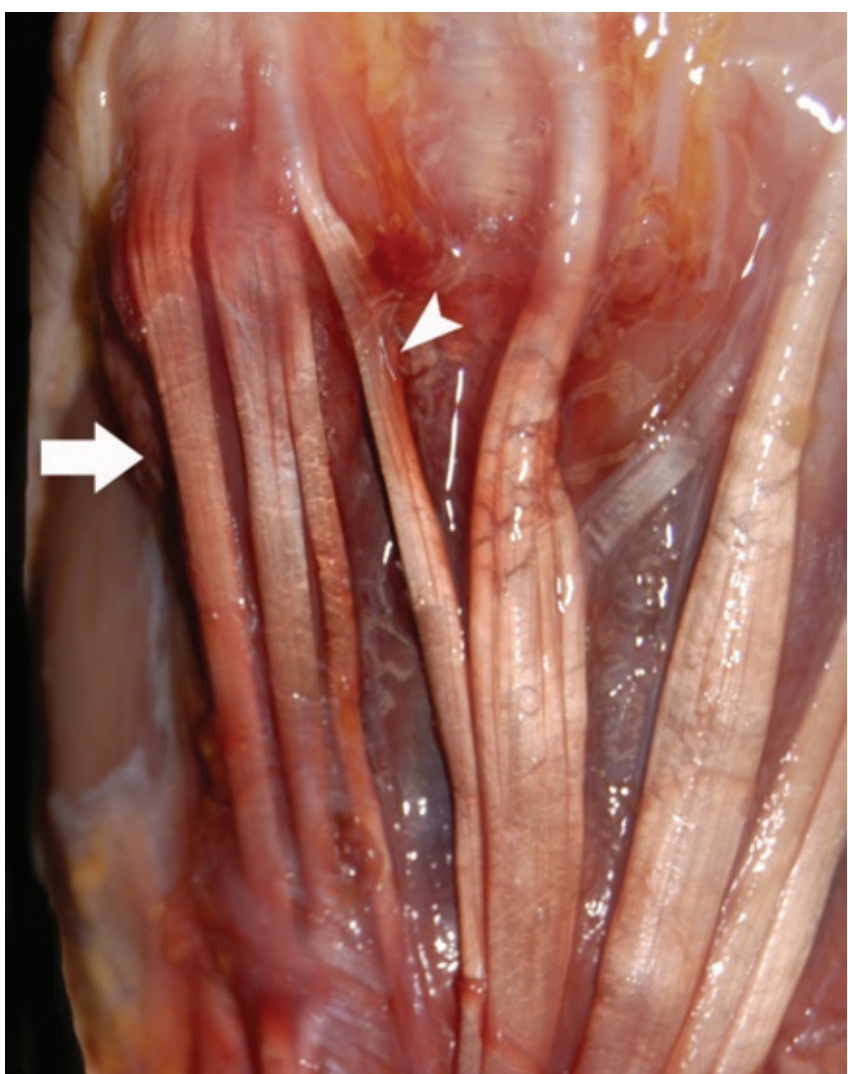

Fig. 3. Dissection of 5th finger of the left hand. Note 4 tendons for this finger (arrow). This is a variation as normally 2 tendons would be expected for this finger

and checkrein ligaments. Our article is based on ultrasound images obtained in volunteers, patients, and anatomical dissections of fresh cadaveric specimens. Ethical board approval was obtained to study the extensor and flexor system of the hand in the cadavers. Good quality images in musculoskeletal (MSK) US of the hand require a high frequency linear transducer and a dedicated MSK system.

\section{Discussion}

\section{Midhand and extensor hood}

At the dorsal midhand, the extensor tendons of the third and fourth finger appear as broad structures with multiple areas of splitting between the fibers that should not be mistaken for pathological fissures (Fig. 1). At the level of the second finger, 2 tendons are present (Fig. 2). The extensor indicis proprius tendon is sometimes used as a potential graft in surgical procedures. At least 2 tendons are also present at the level of the fifth finger. These tendons arise from the 5 th and the 6th compartment (Fig. 3). Proper identification of these multiple tendons in US is important to avoid confusion with longitudinal splits and to confirm the presence of tendons in case they could be used as grafts. The tendons at the midhand are connected by intertendinous bands also termed juncturae intertendinea or connexus ${ }^{(5)}$. These bands are 


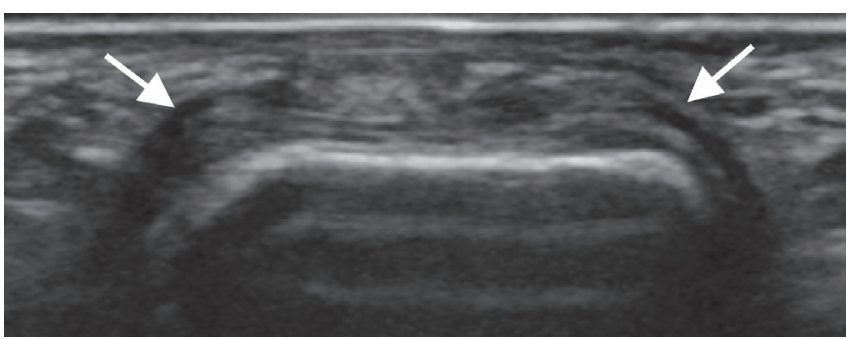

Fig. 4. Coronal US image. 'Dorsal hood' image with extensortendon in midline. The extensor tendon connects to the sides and is enveloped by the sagittal bands (arrows)

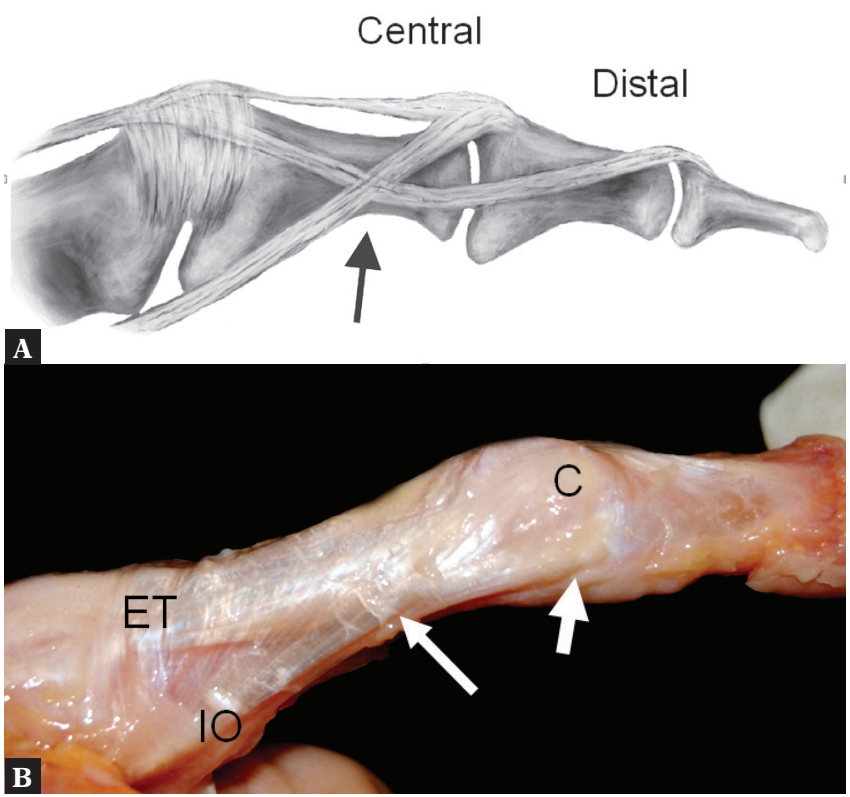

Fig. 5. A. The anatomy of the extensor system is complex. The central extensor tendon continues distally to insert as central slip (central), but gives off fibers to the side that connect to the fibers from the interosseous muscles. Both these continue as the conjoint tendons to form the distal slip insertion (distal). The fibers of the extensor tendon and interosseous tendons mix alongside the proximal phalanx (arrow). B. Note the anatomical dissection showing the central extensor tendon (ET), the interosseous muscles coming from the palmar aspect (IO). They form an $X$ along the side of the phalanx. The conjoint tendons are made up of a slip of the extensor tendon as well as slips of the interosseous and lumbrical muscles. The central tendon inserts as the central slip (C)

classified as A, B, and C (Fig. 1). Type A is located between the 2 nd and 3 th finger and is relatively thin and difficult to visualize. Type B is slightly thicker and located between the 3 th and 4 th finger. Type $\mathrm{C}$ is tendon-like and located between the 4 th and 5 th finger.

The dorsal hood ${ }^{(5)}$ is a complex anatomical structure that serves to keep the extensor tendons centered over the metacarpal heads (Fig. 1). The sagittal bands are the most important components. In the second and fifth fingers, where multiple extensor tendons are present, these tendons are connected to each other. The sagittal bands course alongside the fingers superficial to the collateral ligaments and attach to the palmar plate (Fig. 4). Injuries to the sagittal band usually involve

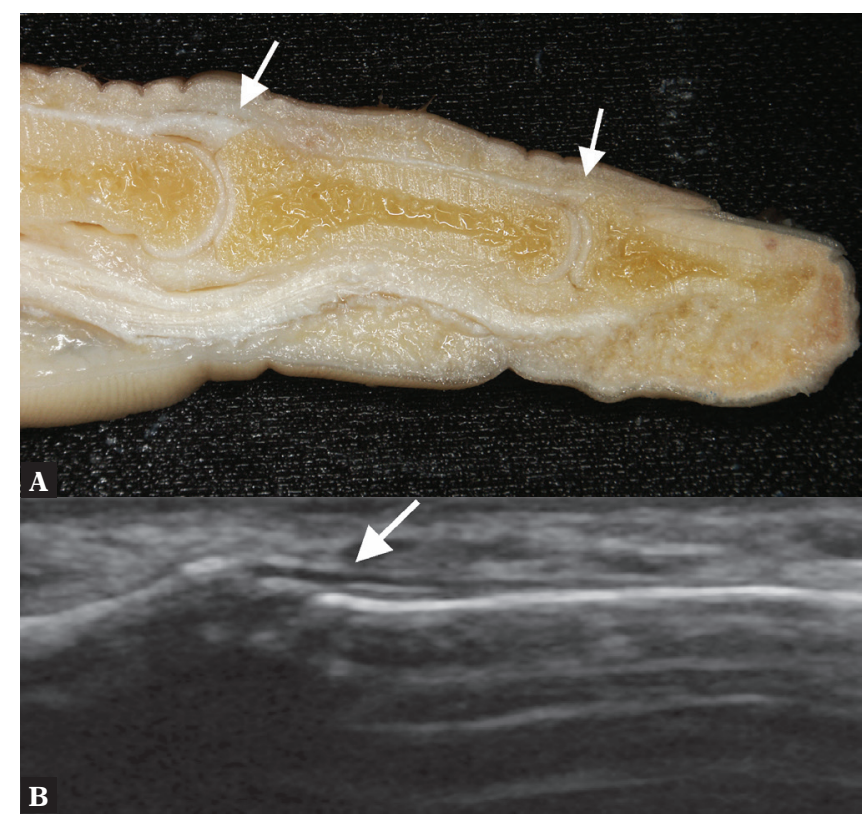

Fig. 6. A. Cadaveric slice showing the central and distal slip insertion (arrows). B. Sagittal US image. The distal slip insertion is smaller and typically more difficult to see (arrow)
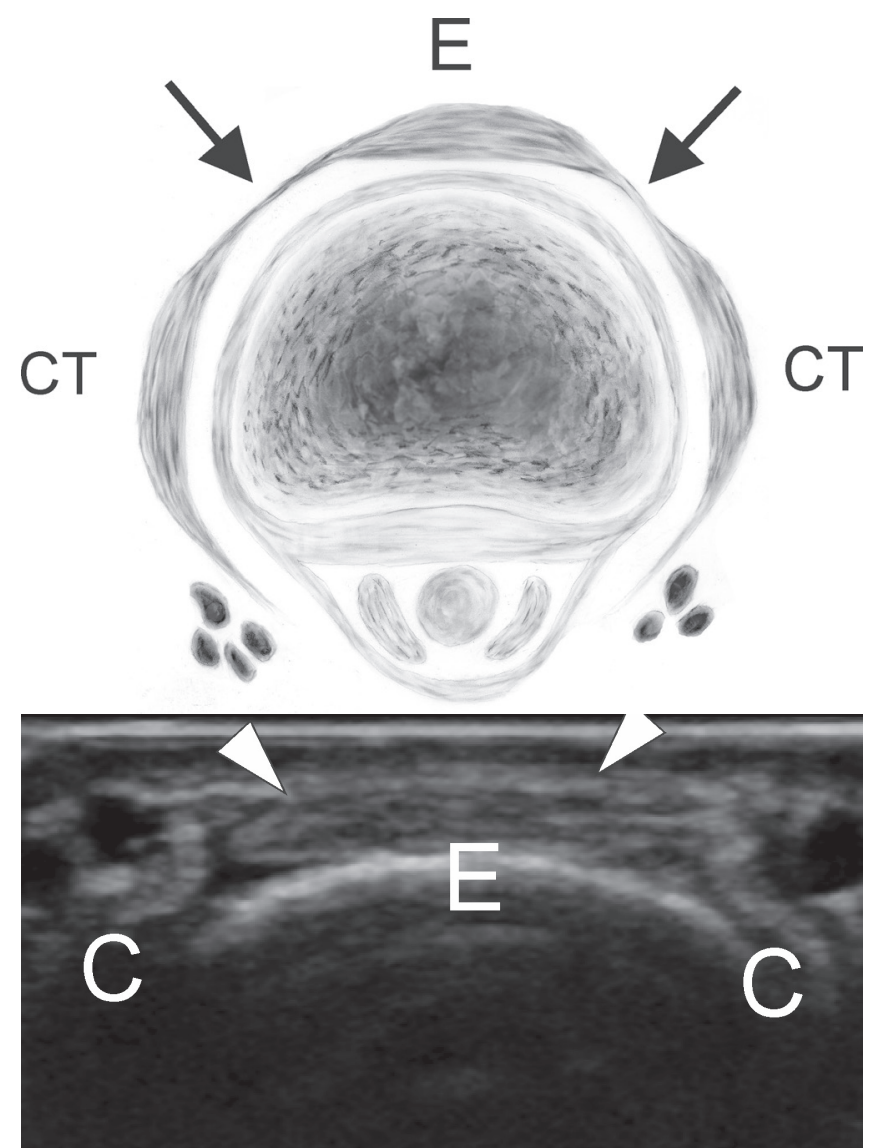

Fig. 7. A. Drawing at the level of the extensor tendon (E) and conjoint tendons $(C T)$. The conjoint tendons are connected by the transverse retinacular ligament (arrows), similar to the dorsal hood. B. Transverse US image shows the conjoint tendons on the side $(C)$ connecting to the extensor tendon (E) by the transverse retinacular ligament (arrowheads) 


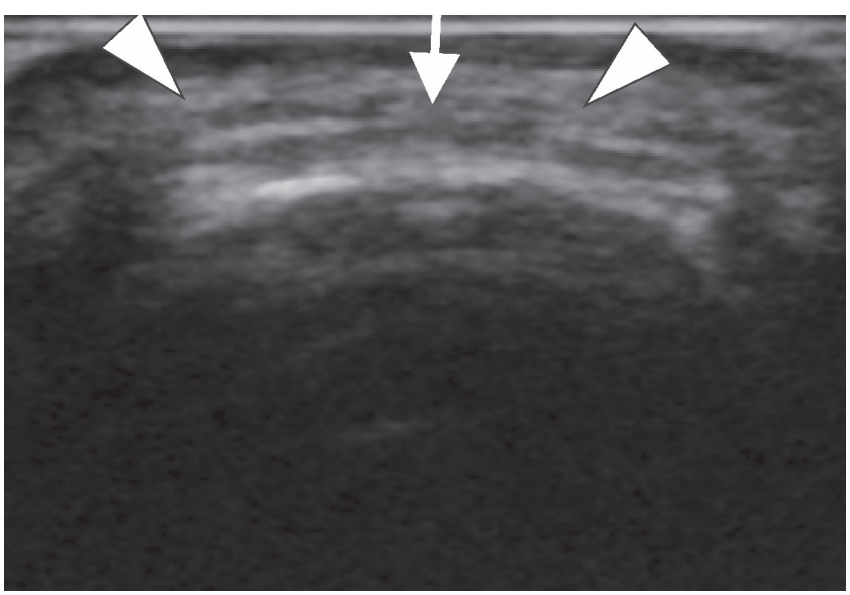

Fig. 8. Transverse image at middle phalanx. The conjoint tendons (arrowheads) continue distally and, before they join, they are connected by the triangular ligament (arrow)

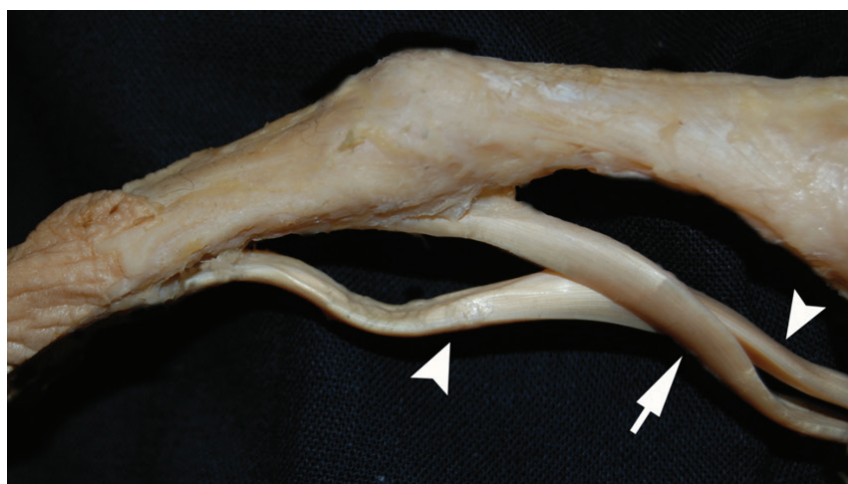

Fig. 9. Dissection showing relationships between flexor profundus (distal) and superficialis (proximal). Note the opening in the flexor superficialis (arrow). The flexor profundus continues distally through this opening (arrowheads). The vincula containing vessels may be present between tendons and tendons and sheath (not shown).

the 3rd finger. The radial band is torn with subluxation of the extensor tendon to the ulnar side. At the 2 nd and 5 th finger, due to the presence of multiple tendons, sagittal band injury may consist of ruptures of the connections between the tendons. Normal thickness of the sagittal bands has been reported to be less than $1.3 \mathrm{~mm}$ in men and less than $0.9 \mathrm{~mm}$ in women. In the case of pathology, the sagittal bands may appear thickened. Distal to the sagittal bands, the dorsal hood continues as an aponeurotic expansion.

\section{Extensor system at the finger}

The extensor anatomy is quite complex ${ }^{(6)}$. The extensor tendon reaches the MCP level, and from this point on, some fibers continue distally and attach to the middle phalanx. Some fibers also descend alongside the finger, where they intermingle with fibers of the interosseous and lumbricalis, coming from the ventral side (Fig. 5A, B, Fig. 6A, B). Some of these fibers continue to the central slip, and some continue alongside the distal phalanx as the conjoint tendons (Fig. 7A, B). The conjoint tendons and the central slip are
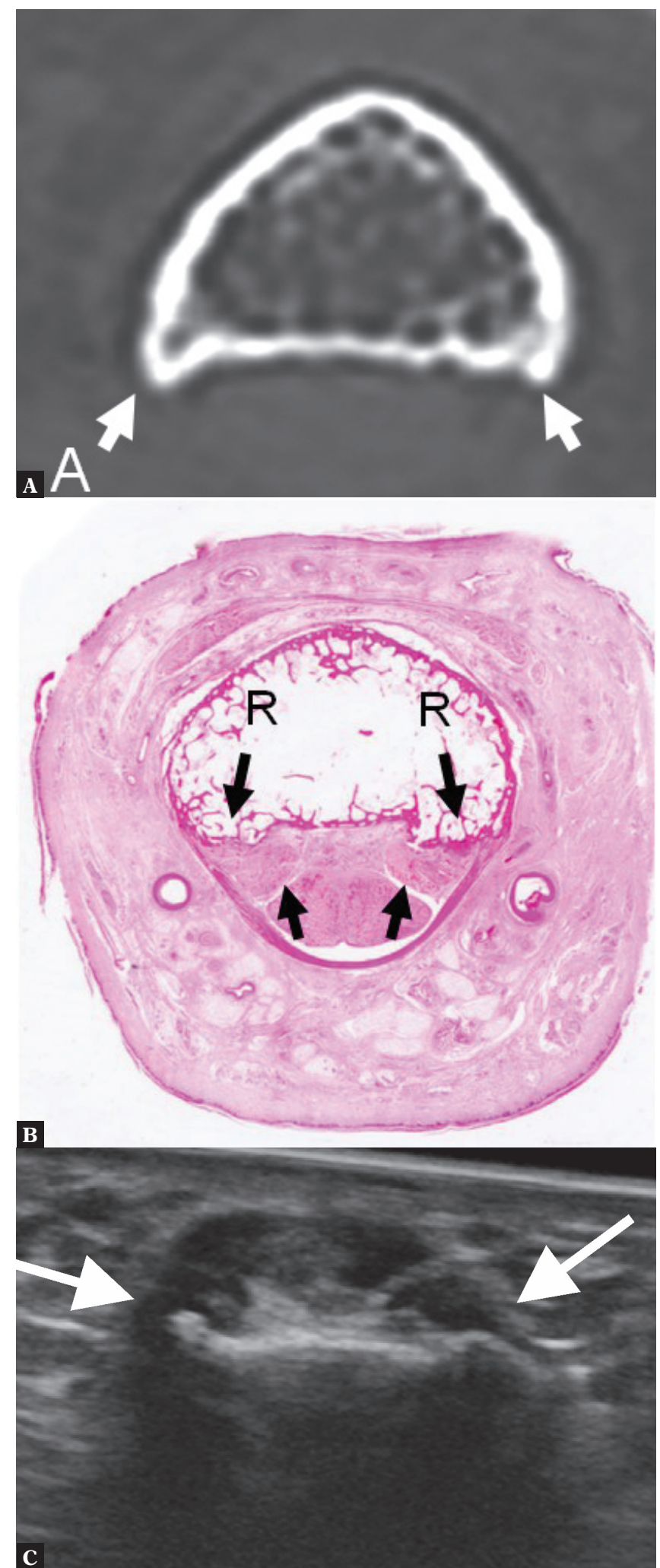

Fig. 10. A. CT scan. Note bone ridges (arrows, A) at the side of the middle phalanx indicating enthesophytes, where the flexor superficialis tendons insert. B. Histological slice showing enthesophytes at the insertion of the flexor superficialis tendons (arrow, $R$, ridges). C. Transverse US image shows flexor superficialis tendon insertions on the phalangeal ridges (arrows) 


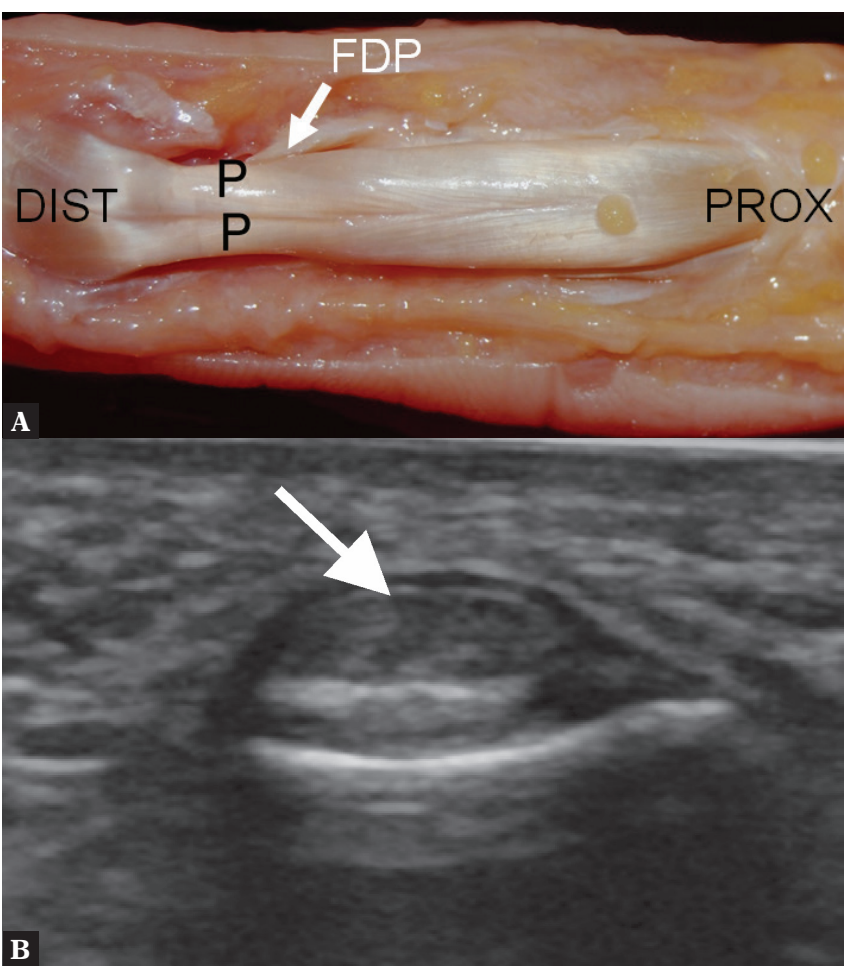

Fig. 11. A. Anatomical dissection. Note that the flexor profundus (FDP, arrow) tendon ( $P$, two parts) appears bifid, which should not be mistaken for a tear. PROX, proximal; DIST, distal B. Transverse US image. Note a central cleft in flexor profundus tendon distally (arrow)

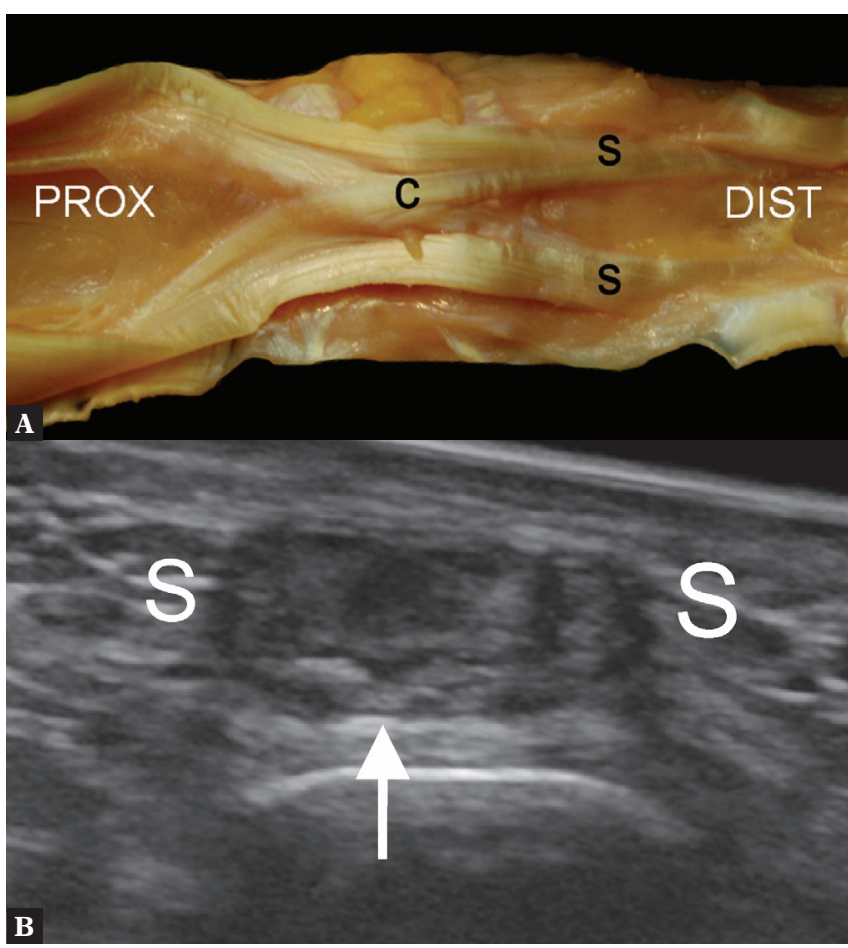

Fig. 12. A. Dissection of the flexor superficialis (s). Note the X-shaped connection between the two tendons deep to the flexor profundus termed the chiasma crurale (c); PROX, proximal, DIST, distal. B. Transverse US shows a connection (arrow) between the flexor superficialis ( $S$, two parts), deep to flexor profundus

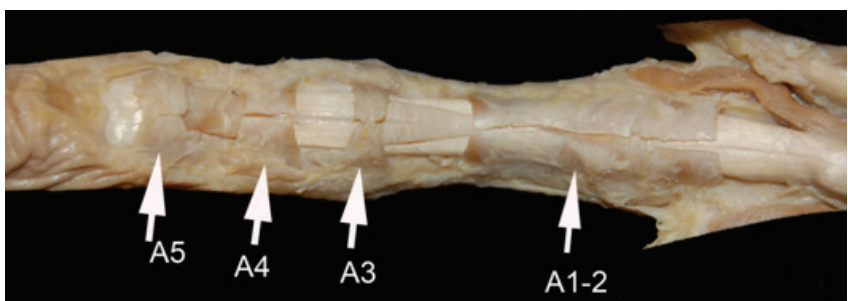

Fig. 13. Dissection of finger. A typical distribution of the annular pulleys (A1 to A5, arrows) is demonstrated

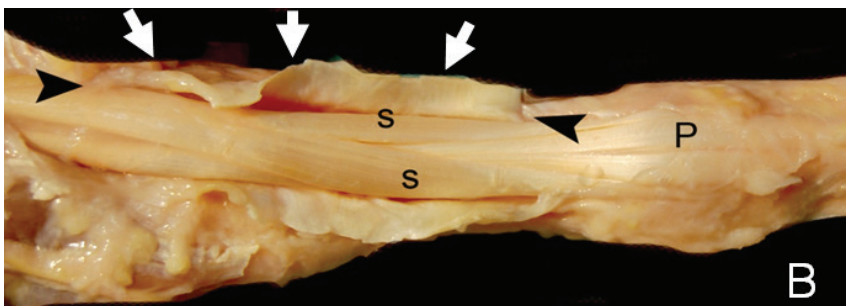

Fig. 14. Dissection of the palmar aspect of finger. An atypical variant of the pulleys. The A1-2-3 pullies (white arrows show sectioned pulley, black arrows extension) are continuous. S, flexor superficialis; P, flexor profundus

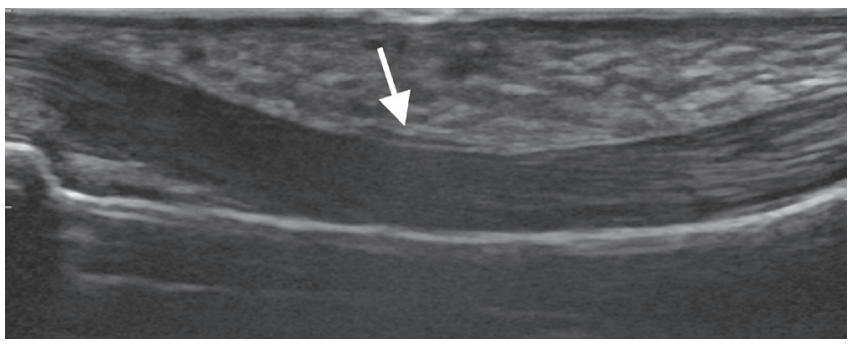

Fig. 15. Sagittal US. Note a thin hypoechoic band covering tendons compatible with A2 pulley (arrow)

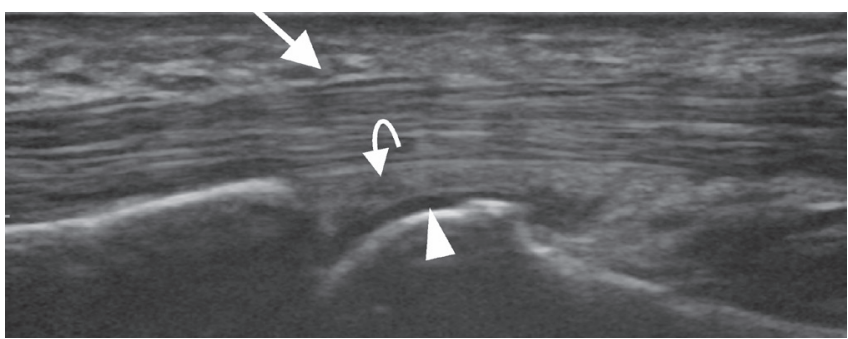

Fig. 16. Sagittal US. Note a thin hypeochoic band covering tendons corresponding to A1 pully (arrow). The arrow head points to cartilage and the curved arrow points to the volar plate

connected by the transverse retinacular ligament, a system similar to the dorsal hood. The central extensor forms the central slip together with contributions from the interosseous and lumbricals. The conjoint tendons continue distally to form the distal slip. Rupture of the central slip typically leads to boutonniere deformity, and rupture of the distal slip results in a mallet finger. Ultrasound allows visualization of the central and distal slip, as well as conjoint tendons, and allows a dynamic examination. Along the midphalanx, a connection is present between the distal extensor tendons, which is known as the triangular ligament (Fig. 8). 


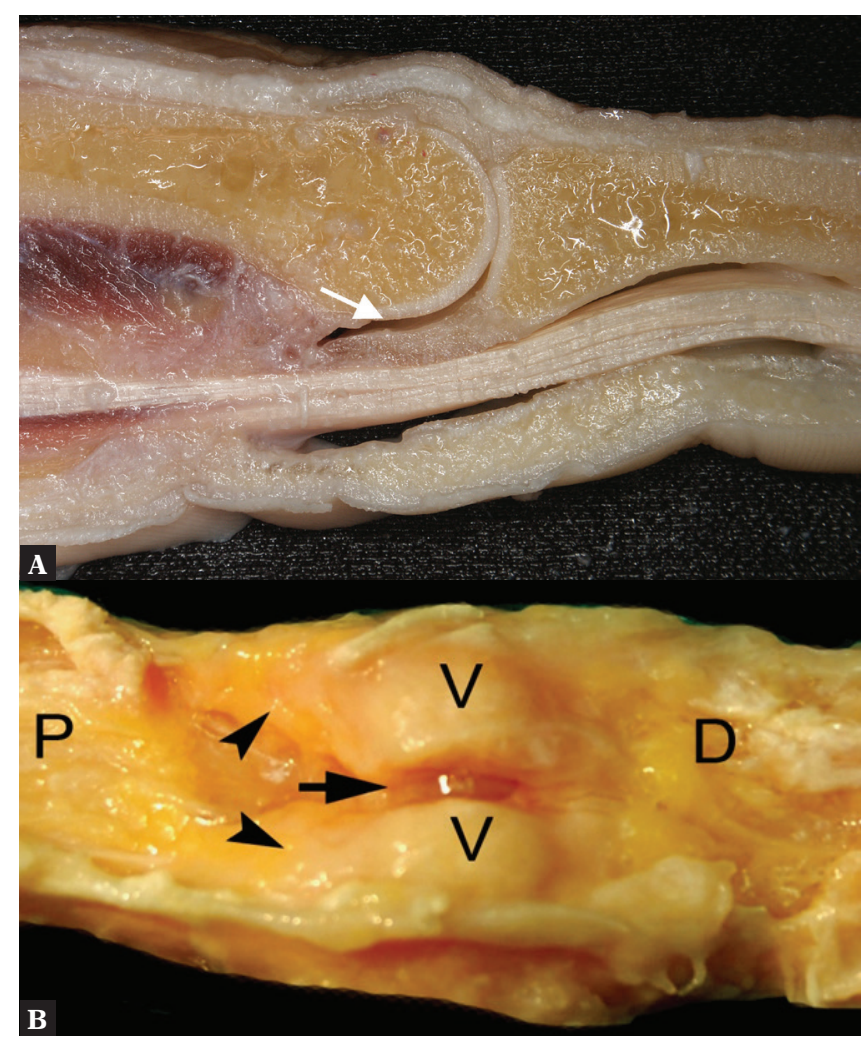

Fig. 17. A. Note the meniscoid palmar plate deep to flexor tendons (arrow). B. Dissection of the palmar-volar plate (v). Note the ridge in the midline which was sectioned (arrow). The arrowheads indicate proximal attachments termed checkrein ligaments; $P$, proximal; $D$, distal

\section{Flexor tendons}

The relationship of the flexor superficialis and flexor profundus varies along the course of these tendons (Fig. 9). The flexor superficialis becomes C-shaped at the level of the proximal phalanx to allow the passage of the flexor profundus. The flexor superficialis then attaches on both sides of the middle phalanx. The flexor profundus then continues distally to insert on the distal phalanx. These anatomical details are well seen on ultrasound, but less on MR imaging. At the insertion of the flexor superficialis on the middle phalanx, ridges may be seen on the bone. These bony ridges ${ }^{(7)}$ represent enthesophytes related to traction of the flexor tendon and the A4 pulley (Fig. 10). The distal continuation of the flexor profundus often shows a central split as a normal variation (Fig. 11). These details are best appreciated on ultrasound and represent a potential pitfall, especially if the tendon is thickened. Before the flexor superficialis inserts on the middle phalanx, connections between both slips arise with an X-shaped appearance (Fig. 12). These connections are thin, but when the tendons are thickened they may become visible and should be identified as such rather than as tears.

\section{Pulley system}

On the palmar aspect of the fingers, pulleys are present that keep the tendons in close proximity to the bone $\mathrm{e}^{(8)}$.

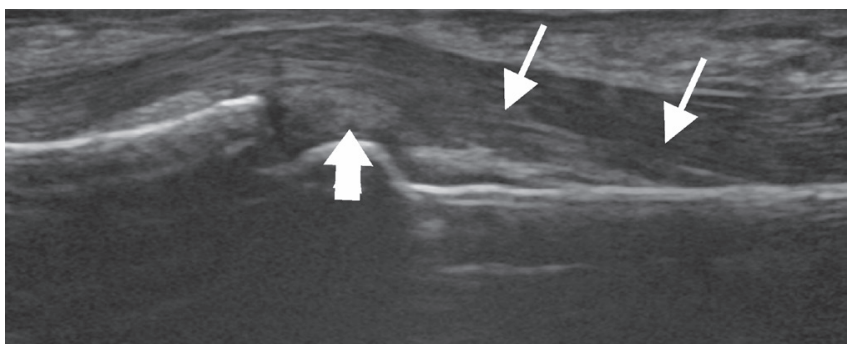

Fig. 18. Note the proximal band-like extension on one side of the volar plate (thick arrow) corresponding to checkrein ligament (arrows)

The annular pulleys are particularly important (Fig. 13). The A2 and A4 pulleys are mechanically most important. The A2 is located at the level of the proximal phalanx, and the A4 at the level of the distal phalanx. The A1, A3, and A5 pulleys are located at the joint levels. When the pulleys are torn, the tendon moves away from the bone, which is called the bowstring injury. Normally, the pulleys are quite thin. All pulleys can be well visualized with state of the art ultrasound equipment. The pulleys may show variations. The A1 and A2 and A3 pulley may be fused instead of presenting as separate pulleys (Fig. 14, Fig. 15). Other variations include a proximal extension of the A1 pulley, sometimes termed A0 pulley. This is clinically important since in the case of the trigger finger the proximal extension should be assessed. A tendon cyst is a common variant on pulleys. This is a misnomer since the cyst is actually located on the pulley and hence does not move with the tendon during a dynamic study. The A2 pulleys insert on ridges on the palmar side of the proximal phalanx. The ridges become more prominent with age.

\section{Volar plate and checkrein ligaments}

The volar plates are fibro-cartilaginous structures having a broad attachment distally. Volar plate avulsion is a common injury (Fig. 16). It appears hyperechoic on ultrasound (Fig. 16). The volar plate contains a central groove (Fig. 17). Proximally, the volar plates attach by thin bands termed the checkrein ligaments. The checkrein ligaments can be better visualized if some joint fluid is present (Fig. 18).

\section{Conclusion}

Ultrasound is an excellent technique to evaluate the flexor and extensor system of the fingers, their variations and closely associated structures. Understanding of the precise anatomy and variants is necessary for adequate image interpretation.

\section{Conflict of interest}

The authors do not report any financial or personal connections with other persons or organizations which might negatively affect the contents of this publication and/or claim authorship rights to this publication. 


\section{References}

1. Chiavaras MM, Jacobson JA, Yablon CM, Brigido MK, Girish G: Pitfalls in wrist and hand ultrasound. AJR Am J Roentgenol 2014; 203: 531-540.

2. Starr HM Jr, Sedgley MD, Means KR Jr, Murphy MS: Ultrasonography for hand and wrist conditions. J Am Acad Orthop Surg 2016; 24: 544-554.

3. Tagliafico A, Rubino M, Autuori A, Bianchi S, Martinoli C: Wrist and hand ultrasound. Semin Musculoskelet Radiol 2007; 11: 95-104.

4. Bianchi S, Martinoli C, Sureda D, Rizzatto G: Ultrasound of the hand. Eur J Ultrasound 2001; 14: 29-34.

5. Kichouh M, De Maeseneer M, Jager T, Marcelis S, Van Hedent E, Van Roy $\mathrm{P}$ et al.: Ultrasound findings in injuries of dorsal extensor hood: correlation with MR and follow-up findings. Eur J Radiol 2011; 77: 249-253.

6. De Maeseneer M, Marcelis S, Jager T, Lenchik L, Pouders C, Van Roy P: Sonography of the finger flexor and extensor system at the hand and wrist level: findings in volunteers and anatomical correlation in cadavers. Eur Radiol 2008; 18: 600-607.

7. Meng J, Willekens I, Cattrysse E, Vereecke E, Geers C, Van Cauteren T et al:: Bony palmar ridges of the phalanges of the human fingers. Surg Radiol Anat 2014; 36: 587-593.

8. McNally EG: Ultrasound of the small joints of the hands and feet: current status. Skeletal Radiol 2008; 37: 99-113. 